\title{
MÉTODO DE POLYA PARA RESOLUÇÃO DE PROBLEMAS MATEMÁTICOS: UMA PROPOSTA METODOLÓGICA PARA O ENSINO E APRENDIZAGEM DE MATEMÁTICA NA EDUCAÇÃO BÁSICA
}

\author{
E.A.S.PONTES ${ }^{*}$ \\ Instituto Federal de Alagoas \\ edelpontes@gmail.com*
}

Artigo submetido em 20/12/2017 e aceito em 24/06/2019

DOI: $10.15628 /$ holos.2019.6703

\section{RESUMO}

No mundo contemporâneo diversas pesquisas são realizadas em busca de uma solução eficaz no processo ensino e aprendizagem de matemática, tendo como foco as suas novas técnicas da educação matemática. Este trabalho tem como objetivo apresentar uma proposta metodológica para o ensino e aprendizagem de matemática na educação básica, através da resolução de problemas utilizando o método de Polya. O método de Polya consiste em três etapas: Compreender o problema, Designar um plano, Executar o plano e
Retrospecto do problema. Metodologicamente serão apresentados três problemas matemáticos cuja resolução seguirá o método de Polya. Está sugestão, Resolução de Problemas através do método de Polya, como prática educacional no processo de ensino e aprendizagem de matemática possibilita ao professor facilitador e ao aluno aprendiz desenvolver novas habilidades no intuito de fortalecer o pensamento crítico e o raciocínio lógico.

PALAVRAS-CHAVE: Ensino e aprendizagem de matemática, método de Polya, Resolução de problemas.

\section{METHOD OF POLYA FOR THE SOLUTION OF MATHEMATICAL PROBLEMS: A METHODOLOGICAL PROPOSAL FOR TEACHING AND LEARNING OF MATHEMATICS IN BASIC EDUCATION}

\begin{abstract}
In the contemporary world several researches are carried out in search of an effective solution in the teaching and learning process of mathematics, focusing on their new techniques of mathematical education. This work aims to present a methodological proposal for the teaching and learning of mathematics in basic education, through problem solving using the Polya method. The Polya method consists of three steps: Understanding the problem, Designating a plan, Performing the plan, and Retrospection the problem.
\end{abstract}

\begin{abstract}
Methodologically, three mathematical problems will be presented whose resolution will follow the Polya method. This suggestion, Problem Solving through the Polya method, as an educational practice in the teaching and learning process of mathematics enables the facilitator teacher and the learner to develop new skills in order to strengthen critical thinking and logical reasoning.
\end{abstract}

KEYWORDS: Teaching and learning of mathematics, Polya method, Problem solving. 


\section{INTRODUÇÃO}

No mundo contemporâneo diversas pesquisas são realizadas em busca de uma solução eficaz no processo ensino e aprendizagem de matemática, tendo como foco as suas novas técnicas da educação matemática. Os conteúdos de matemática na educação básica devem passar por um criterioso processo de reformulação e deve ser repensado através destas novas técnicas apresentadas na educação matemática. Para Oliveira e Calejon (2016) a educação matemática é um movimento reflexivo de suma importância para repensar as condições de ensino dos conteúdos de matemática, de maneira que se possa diminuir a aversão que essa ciência produz.

A sociedade está em constante evolução e transformação e a prática docente caminha em sentido oposto acreditando em soluções conservadoras, metódicas e ultrapassadas. Enquanto os professores repassam seus conhecimentos no quadro negro ou utilizam livros didáticos com exercícios e atividades, muitas vezes sem uma relação com o cotidiano, seus alunos acessam de forma instantânea seus celulares e computadores com respostas prontas para suas indagações e inquietudes. Não devemos acreditar que essas máquinas substituirão, em pouco tempo, o professor, ser pensante, criativo e racional, e sim, acreditar em criar meios para minimizar as defasagens entre o que se ensina e o que realmente se usa no dia a dia. Da Silva et al (2017) comenta que é preciso criar ambientes instigadores para o ensino de matemática, de forma que os alunos possam produzir matemática e compreender todo o processo de construção dos conceitos matemáticos.

A sala de aula se torna ambiente agradável quando se apresenta práticas motivadoras e criativas com perfeita sintonia com o mundo moderno, recheado de indivíduos de raciocínio lógico apurado, intuitivos e de pensamento matemático aumentado. O mundo tecnológico e a matemática se confundem por haver uma relação biunívoca entre o modelo criador e o ser criado. O ensino tradicional, dos conteúdos de matemática, deve ser substituído por um ensino motivador aproximando o aluno da sua realidade, essas mudanças devem-se essencialmente ao surgimento da era computacional.

E aí surge o primeiro e grande desafio: como devemos ensinar nossas crianças se nossos educadores não estão aptos para entender as novas tecnologias? Para que exista uma relação prazerosa da criança com a escola que frequenta, é necessário que nossos professores possam criar meios de aproximação entre ele e seu aprendiz. "Em Matemática, os professores devem buscar tópicos relacionados com as situações vivenciadas no dia a dia e incentivar os alunos a desenvolverem seus próprios métodos de resolução de problemas" (Nunes Otaviano; Lima Soriano de Alencar; Fukuda, 2012, p. 62).

O método de resolução de problemas matemáticos tem sido amplamente estudado e discutido na comunidade de Educação Matemática. Apesar de haver uma preocupação internacional expressa nas novas propostas curriculares voltadas para a educação básica e de formação de professores, observamos que na prática esta metodologia ainda é pouco 
explorada tanto nos cursos de formação inicial para professores de matemática, quanto nas salas de aulas da educação básica no Brasil. (Carvalho \& Civardi, 2012, p.726).

Para Lester (1994) existem três razões encontradas para os estudantes não serem capazes de resolver problemas: (1) a resolução de problemas é uma atividade intelectual extremamente complexa; (2) há falta de saber dos envolvidos do que é necessário para a resolução de problemas; e (3) são dadas muito poucas oportunidades aos alunos para se envolverem realmente na resolução de problemas.

Segundo Schoenfeld (1996) o pensar matematicamente, a partir de resolução de problemas, significa: (1) conseguir enxergar o mundo de um ponto de vista matemático, isto é, aplicar ideias matemáticas em diversas situações, e (2) ter as ferramentas de oficio para matematizar com sucesso.

De acordo com os Parâmetros Curriculares Nacionais (BRASIL, 1998) o processo de ensino e aprendizagem na matemática se desenvolve de forma mais proveitosa a partir da utilização de propostas de resolução de problemas matemáticos. Segundo De Jesus Costa e Freitas (2017) a resolução de problemas contribui para o desenvolvimento da leitura, da criatividade e do raciocínio lógico, permitindo maior autonomia e autoconfiança para o aprendiz.

A criança ela deve estar apta a desenvolver, intuitivamente, meios de fugir das sequências pré-determinadas e obrigatórias em seu meio escolar. Em contrapartida, faz-se necessário a utilização de pensamentos mais lógicos e criativos. Um problema matemático bem elaborado representa no processo ensino-aprendizagem um componente pedagógico fundamental para a produção de conhecimento. Por meio da resolução de problemas, a criança atiça sua curiosidade e desenvolve a arte de criar e ampliar seu meio de convívio. Desta forma, nosso trabalho tem como objetivo apresentar uma proposta metodológica para o ensino e aprendizagem de matemática na educação básica, através da resolução de problemas utilizando o método de Polya.

\section{NOVAS TECNOLOGIAS EM MATEMÁTICA: RESOLUÇÃO DE PROBLEMAS}

As transformações científicas e tecnológicas que ocorreram nas últimas décadas acenderam um sinal de alerta para uma grande parcela de educadores teóricos e resistentes a mudança na forma de ensinar matemática. O conceito de matemática vem do latim mathematǐca, que podemos traduzir como conhecimento: matemática é a ciência dos padrões, ciência absoluta, factual, dedutiva e se dedica as propriedades e relações abstratas, através de números, símbolos e figuras geométricas.

No contexto atual, o ensino de matemática na educação básica requer uma quebra de paradigmas na sua forma de apresentar seus conteúdos. As novas formas para o ensino e aprendizagem eficiente de matemática surgiram para melhorar o entendimento desta ciência. Uma dessas formas é a resolução de problemas de matemática, tema deste trabalho. Para Polya 
(1995) resolver um problema significa encontrar um caminho que ainda não é conhecido e que contorne um obstáculo para alcançar o objetivo traçado, por meios adequados.

Para Zuffi e Onuchic (2007) alguns aspectos devem ser estimulados em um processo de ensino e aprendizagem através da resolução de problemas: (1) compreender os dados de um problema, (2) tomar decisões para resolvê-lo, (3) estabelecer relações, (4) saber comunicar resultados, e (5) ser capaz de usar técnicas conhecidas.

Diversas abordagens dos conteúdos de matemática na educação básica podem ser tratadas a partir da resolução de problemas, de maneira, que leve a criança a compreender melhor o tema proposto e consequentemente desenvolver o raciocínio lógico e sua criatividade. A proposta não é apenas de encontrar a solução do problema, e sim, acompanhar todo o processo de construção desta solução. Para Maia e Proença (2016) saber diferenciar o que é um problema de um exercício é uma das práticas mais comuns e de maior dificuldade para o professor. Um verdadeiro problema deve se constituir um real desafio em que os alunos, por meios de sequencias de ações, buscarão obter os resultados.

Onuchic e Allevato (2011) afirmam que no processo de ensino, aprendizagem e avaliação de matemática a partir da resolução de problemas, o problema é o ponto de partida, deste modo, por meio da resolução de problemas, os alunos são habilitados a fazer diversas conexões nos conteúdos de matemática, gerando novas propostas e novos conceitos.

Quando pensamos em problemas matemáticos, de imediato, nos vêm a mente diversas fórmulas para tentarmos encontrar a solução do problema proposto. É importante frisar que o fazer matemática está diretamente relacionada com a prática de resolver problemas, e muitas vezes essas fórmulas nos dão praticidade para gerarmos a solução correta. Para de Medeiros $(2001$, p.2):

Um rápido olhar sobre o desenvolvimento do conhecimento matemático, ao longo do tempo, nos leva a perceber que a atividade de resolução de problemas lhe serve de motor. No entanto, o trabalho com resolução de problemas, em sala de aula, no Ensino Fundamental, não está tendo, para a aprendizagem da matemática um papel que, ao menos, se aproxime daquele desenvolvido nesse campo do conhecimento. [...] De modo geral, os problemas são trabalhados em sala de aula para "fixar" os assuntos que acabaram de ser estudados. Eles se caracterizam como exercícios repetitivos, permitindo ao aluno identificar certas características que se repetem no processo de resolução, criando procedimentos padronizados para serem utilizados na resolução de problemas semelhantes.

O currículo de matemática na educação básica deve está sintonizado com as necessidades de nosso mundo tecnológico, exatamente pelo fato que a criança do século XXI está intuitivamente relacionada e adaptada às novas tecnologias. A utilização de resolução de problemas nas séries iniciais da educação básica terá um papel fundamental no processo ensino e aprendizagem de matemática, pois induzirá a criança a pensar, calcular e tomar decisão. 


\section{MÉTOdO DE POLYA PARA RESOLUÇÃO DE UM PROBLEMA DE MATEMÁTICA}

O principal objetivo de nosso trabalho é apresentar uma forma eficiente do professor ensinar e do aluno aprender matemática a partir de resolução de problemas.

Segundo Polya (1995) existem quatro fases para resolver um problema de matemática de forma eficiente:

$\checkmark$ Compreender o problema (CP): o que é necessário para resolvê-lo? Quais suas variáveis e incógnitas?

$\checkmark$ Designar um plano (DP): Esse problema é conhecido? Como as variáveis estão correlacionadas? Quais estratégias devemos usar para sua resolução?

$\checkmark$ Executar o plano (EP): é possível verificar cada passo da execução? É possível demonstrar que o plano está correto?

$\checkmark$ Retrospecto do problema (RP): é possível verificar o resultado encontrado?

Percebe-se que este processo de resolução de um problema de matemática proposto por Polya é um método bastante interessante e bem planejado e se for bem executado nos dará os resultados esperados.

$$
\mathrm{CP} \rightarrow \mathrm{DP} \rightarrow \mathrm{EP} \rightarrow \mathrm{RP}
$$

A compreensão do problema é o ponto inicial do processo e se faz necessário que o aprendiz interprete o enunciado do problema e tenha vontade de resolvê-lo. Em seguida, é preciso estabelecer um plano para a resolução do problema, apontando suas variáveis, suas hipóteses e seus modelos. A execução do plano só será eficaz se todo o planejamento desde da sua compreensão até as estratégias a seguir forem realizadas plenamente. Por fim, fazer uma retrospectiva do problema executado é de total valia, pois o mesmo comprovará a verdade do resultado encontrado.

\section{RESOLUÇÃO DE PROBLEMAS PELO MÉTODO DE POLYA}

$\mathrm{O}$ ato de ensinar matemática na educação básica deve oportunizar condições para a criança gerar autonomia suficiente para estabelecer relações dos conteúdos aprendidos com os modelos de seu dia a dia. Pontes (2018) afirma que a grande discussão no processo de ensinar e aprender matemática é saber quais os indivíduos, aluno ou professor, que possuem habilidades e competências para melhor abstrair os conceitos e relações de matemática.

O procedimento mecânico de memorização deve ser substituído por métodos criativos e de raciocínio lógico, de tal forma que o aluno esteja motivado e pronto para desenvolver seus conhecimentos e saberes. Desta forma, apresentaremos nesta seção três problemas matemáticos seguindo as etapas do método de Polya.

Problema 01: (JONOFON, 2004) Um casal tem filhos e filhas. Cada filho tem o número de irmãos igual ao número de irmãs. Cada filha tem o numero de irmãos o dobro do número de irmãs. Qual é o total de filhos e filhas do casal? 
Seguindo o algoritmo de Polya, teremos:

CP: O problema consiste em determinar o número de filhos e filhas de um casal, seguindo as hipóteses apresentadas. Desta forma, seja $x$ o número de filhos e $y$ o número de filhas.

DP: O plano será estabelecido seguindo as seguintes hipóteses: Se $x$ é o número de filhos, então o número de seus irmãos será $x-1$. Se $y$ é o número de filhas, então o número de suas irmãs será $y-1$. Assim, cada filho tem $x-1$ irmãos e $y$ irmãs e cada filha tem $y-1$ irmãs e $x$ irmãos.

EP: Observa-se que se seguirmos o plano estabelecido e atentando para o enunciado do problema, podemos formar o seguinte sistema:

$\left\{\begin{array}{c}x-1=y \\ x=2(y-1)\end{array}\right.$. Resolvendo o sistema de equações teremos: $x=4$ e $y=3$.

RP: Substituindo os resultados encontrados no modelo, verificamos que os valores são compatíveis. Logo, o casal tem um total de 7 filhos.

O problema sobre idades (Problema 01) sua resolução parte da ideia de gerar duas incógnitas $(x$ e $y$ ) e estabelecer um sistema de equações com essas variáveis pré-determinadas. 0 método de Polya é extremamente didático e de fácil compreensão e leva a criança aprendiz a acompanhar passo a passo o modelo desejado.

Problema 02: (JONOFON, 2004) Um rato está 48 metros na frente de um gato que o persegue. Enquanto o rato percorre 4 metros o gato percorre 7 metros. Quantos metros deverá percorrer o gato para alcançar o rato?

Seguindo o método de Polya, teremos:

CP: Este é um problema de perseguição entre um gato e um rato e consiste em determinar quantos metros deverá percorrer o gato para alcançar o rato.

DP: O plano proposto será seguindo as hipóteses apresentadas:

Hipótese 1: A distância entre o gato e o rato é de 48 metros.

Hipótese 2: Enquanto o rato percorre 4 metros, o gato percorre 7 metros.

A cada 7 metros percorridos pelo gato, o rato percorre apenas 4 metros, essa vantagem do gato a cada 7 metros é de $7 m-4 m=3 m$.

EP: Nota-se que se seguirmos o plano estabelecido e atentando para o enunciado do problema, podemos gerar as seguintes indagações:

O gato terá que percorrer7 metros tantas vezes, quantos 3 metros existir em 48 metros, isto é, $\frac{48 \mathrm{~m}}{3 \mathrm{~m}}=16$ vezes. Portanto, o gato terá que percorrer 16 vezes 7 metros, logo $16 x 7 \mathrm{~m}=112$ metros.

RP: Percebe-se que o resultado encontrado está compatível com o enunciado do problema. Logo, o gato terá que percorrer 112 metros para alcançar o rato. 
Este problema de perseguição entre animais (Problema 02) é bastante clássico e aparece na maioria dos livros de matemática e sua resolução requer bastante atenção na sua estrutura e raciocínio lógico. O método de Polya, para esse caso, se apresenta de forma didática e organizada e a criança poderá desenvolver toda sua criatividade e raciocínio para solucionar o problema.

Problema 03: (JONOFON, 2004) Um percurso a ser feito por um ciclista foi dividido em quatro partes de comprimentos iguais. Na primeira parte, de estrada plana, fez $10 \mathrm{~km}$ por hora, na segunda, uma subida, fez $5 \mathrm{~km}$ por hora, na terceira, uma descida, fez $30 \mathrm{~km}$ por hora, na última, de estrada plana, mas com vento nas costas, fez $15 \mathrm{~km}$ por hora. Qual a velocidade média do ciclista no percurso?

Seguindo o método de Polya, teremos:

CP: Este é um problema de física e consiste em determinar a velocidade média de um ciclista em um determinado percurso. Suponhamos que o aprendiz conhece a relação $V=\frac{S}{t}$, onde $V$ é a velocidade, $S$ é o espaço e $t$ o tempo. Designemos $S$ o espaço que o ciclista percorreu em cada parte do percurso. Desta forma, o percurso total será de 4S.

DP: O plano proposto será apresentar o tempo que o ciclista gastou para percorrer cada parte do percurso:

A primeira parte: $t_{1}=\frac{S}{10}$; a segunda parte: $t_{2}=\frac{S}{5}$; a terceira parte: $t_{3}=\frac{S}{30}$ e a quarta parte: $t_{4}=\frac{S}{15}$.

EP: Observa-se que através do plano estabelecido e atentando para o enunciado do problema, temos:

O tempo total gasto pelo ciclista para percorrer o percurso é dado por: $\frac{S}{10}+\frac{S}{5}+\frac{S}{30}+\frac{S}{15}=$ $\frac{2 S}{5}$. Como a velocidade média é a razão da distância percorrida (percurso total) pelo tempo gasto no percurso, então, teremos: $V_{m}=\frac{4 S}{\frac{2 S}{5}}=10 \mathrm{~km} / \mathrm{h}$.

RP: Percebe-se que o resultado encontrado está compatível com o enunciado do problema. Logo, a velocidade média do ciclista no percurso é de $10 \mathrm{Km} / \mathrm{h}$.

Este problema envolvendo distâncias, tempos e velocidades (Problema 03) durante bastante tempo atormentou estudantes, principalmente aqueles que não tiveram conhecimento de mecânica. Os tópicos de física apresentados neste problema podem ser desenvolvidos para crianças da educação básica desde que elas tenham conhecimento sobre aritmética. $O$ método de Polya, para esse caso, se apresenta de forma bastante interessante e vai facilitar o aprendizado da criança para solucionar o problema.

\section{CONSIDERAÇÕES FINAIS}

A sociedade da informação e do conhecimento exige que os indivíduos estejam aptos a enfrentar os desafios do mundo moderno e estejam prontos a tomar decisões coerentes e 
eficientes. O conhecimento matemático torna-se, desta forma, imprescindível para a geração de modelos eficazes na explicação dos fenômenos da natureza.

Diante do exposto, pode-se fazer uma reflexão geral sobre a importância da resolução de problemas, particularmente utilizando o método de Polya, no processo de ensino e aprendizagem de matemática. É conveniente salientar que todos os procedimentos adotados se apresentam como uma sugestão para orientar o aluno a seguir passo a passo a construção de novos conhecimentos e saberes.

Utilizando-se o método proposto por Polya constata-se que, com mais facilidade, organizam-se as ideias e se obtém a solução do problema com uma melhor compreensão do que se não tivéssemos seguido seu método. Também é possível encontrar problemas análogos e tornar mais clara uma estratégia para sua resolução. Certamente esse método não é uma ferramenta milagrosa, mas torna-se necessário e eficiente seu uso em um grande número de problemas, principalmente os que apresentam um maior nível de dificuldade (Gazzoni \& Ost, 2008, p.44)

O tema resolução de problemas através do método de Polya, como prática educacional no processo de ensino e aprendizagem de matemática possibilita ao professor facilitador e ao aluno aprendiz desenvolver novas habilidades no intuito de fortalecer o pensamento crítico e o raciocínio lógico. Espera-se que outras experiências possam ser sugeridas como modelos transformadores de uma educação pautada no ser engenhoso, produtivo e que esteja pronto para enfrentar os desafios do século XXI.

\section{REFERÊNCIAS}

Carvalho, F. D. P. S., \& Civardi, J. A. (2012). Novas tecnologias, velhas atitudes, práticas antigas. In II Congresso Internacional TIC e Educação.

D’Ambrosio, B. S. (1989). Como ensinar matemática hoje. Temas e debates, 2(2), 15-19.

Gazzoni, A., \& Ost, A. (2008). A resolução de um problema: soluções alternativas e variações na formulação. VIDYA, 28(2), 10.

Jesus Costa, G. C., \& Freitas, A. V. (2017). ANÁLISE DE ESTRATÉGIAS DE RESOLUÇÃO DE PROBLEMAS MATEMÁtICOS DE ALUNOS DA EJA. Revista de Educação, Ciências e Matemática, 7(2).

Lester, F. (1994). O que aconteceu à investigação em resolução de problemas de Matemática? A situação nos Estados Unidos. Resolução de problemas: Processos cognitivos, concepções de professores e desenvolvimento curricular, 13-31.

Maia, É. J., \& de Proença, M. C. (2016). A resolução de problemas no ensino da geometria: dificuldades e limites de graduandos de um curso de pedagogia. Revemat: Revista Eletrônica de Educação Matemática, 11(2), 402-417. 
Medeiros, K. M. (2001). O contrato didático e a resolução de problemas matemáticos em sala de aula.

Nunes Otaviano, A. B., Lima Soriano de Alencar, E. M., \& Fukuda, C. C. (2012). Estímulo à criatividade por professores de Matemática e motivação do aluno. Psicologia Escolar $e$ Educacional, 16(1).

Oliveira, S. G., \& Calejon, L. M. C. (2016). O JOGO TORRE DE HANÓI PARA O ENSINO DE CONCEITOS MATEMÁTICOS. Revista de Ensino de Ciências e Matemática, 7(4), 149-158.

Onuchic, L. D. L. R., \& Allevato, N. S. G. (2011). Pesquisa em Resolução de Problemas: caminhos, avanços e novas perspectivas. Bolema-Mathematics Education Bulletin, 73-98.

Polya, George. (1995). A arte de resolver problemas: um novo aspecto do método matemático. Trad. Heitor Lisboa de Araújo. 2a reimpressão. Rio de Janeiro.

Pontes, E. A. S. (2013). HIPERMAT-Hipertexto Matemático: Uma ferramenta no ensinoaprendizagem da matemática na educação básica. Psicologia \& Saberes, 2(2).

Pontes, E. A. S. (2017). Os números naturais no processo de ensino e aprendizagem da matemática através do lúdico. Diversitas Journal, 2(1), 160-170.

Pontes, E. A. S. (2018). A ARTE DE ENSINAR E APRENDER MATEMÁTICA NA EDUCAÇÃO BÁSICA: UM SINCRONISMO IDEAL ENTRE PROFESSOR E ALUNO. Revista Psicologia \& Saberes, 7(8), 163-173.

Schoenfeld, A. (1996). Porquê toda esta agitação acerca da resolução de problemas. Investigar para aprender matemática, 61-72.

Sérates, Jonofon (2004). Raciocínio lógico. Brasília: Jonofon Sérates, 11. ed. v.2.

Silva, A. J. N., do Nascimento, A. M. P., \& Muniz, C. A. (2017). O Necessário Olhar do Professor sobre a Produção Matemática das Crianças nos Anos Iniciais. Educação Matemática em Revista, 48-55.

Zuffi, E. M., \& ONUCHIC, L. D. L. R. (2007). O ensino-aprendizagem de matemática através da Resolução de Problemas e os processos cognitivos superiores. Revista iberoamericana de educación matemática, (11), 79-97. 